\title{
Mathematical Formulation for the AlliSya Protection Plus Integration Model
}

\author{
Rini Cahyandari ${ }^{1}$, Asep Solih Awalluddin ${ }^{2}$, Dwi Putri Utami ${ }^{3}$, Tika Karlina Rachmawati ${ }^{4}$ \\ Wahyudin Darmalaksana ${ }^{5}$, Sukono $^{6}$, Puspa Liza Ghazali ${ }^{7}$ \\ \{rini_cahyandari@uinsgd.ac.id ${ }^{1}$ \} \\ Department of Mathematics, UIN Sunan Gunung Djati Bandung, Indonesia ${ }^{1,2,3}$ \\ Department of Mathematics Education, UIN Sunan Gunung Djati Bandung, Indonesia ${ }^{4}$ \\ Department of Hadits Science, UIN Sunan Gunung Djati Bandung, Indonesia ${ }^{5}$ \\ Department of Mathematics, Universitas Padjadjaran, Indonesia ${ }^{6}$ \\ Department of Finance, Universiti Sultan Zainal Abidin, Malaysia ${ }^{7}$
}

\begin{abstract}
AlliSya Protection is a unit link sharia life insurance product that offers two benefits at once, that is the benefits of protection and investment. Some additional benefits are also offered by this product in the form of the risk of critical illness, disability, hospitalization, accidents, and premium exemption. The explanation of unit link sharia insurance product is generally presented in the form of summary illustrations that can be varied in shape and many pages. In this paper will be discussed about the integration model as an alternative presentation form of summarizing illustrations of unit link sharia insurance, namely AlliSya Protection Plus. Through the mathematical formulation written in each column of the model integration table, will be seen the premium fund management scheme of unit link sharia which is more clearly, sequentially and easily readable.
\end{abstract}

Keywords: Unit Link Sharia Life Insurance, Integration Model, Mathematical Formulation, Premium Fund Management Scheme

\section{Introduction}

Sharia link unit is a sharia insurance product that combines insurance with investment. If someone becomes a customer of this product, there will be two benefits at once, namely insurance and investment protection [18]. Similarly in premium payments, there is a portion of protection premium payments and a portion of investment premium payments. Payment of protection premiums is the same as payment of insurance premiums in general, while the next payment of investment premiums will be managed by the company for investment units in certain products. If the investment result is profitable and can cover the premium costs, then the customer has the option to not pay the premiums in a certain period.

AlliSya Protection Plus is one of life in insurance products combined with sharia-based investments. This insurance product offers basic benefits in the form of life insurance and additional benefits in the form of money insurance for the risk of critical illness, disability, hospitalization, accidents and premium exemptions [20]. Illustrations of AlliSya Protection Plus are presented in several sheets containing information about the amount of premiums, summary of benefits, summary of transactions, death compensation illustrations and other 
information. In this paper will be discussed about the integration model as an alternative illustration presentation of AlliSya Protection Plus, in the form of table.

\section{Methodology}

The figure 1 below is a scheme of managing premium funds for life insurance unit link sharia AlliSya Protection Plus [20]. This scheme is information that must be conveyed to customers, so that the excellence of sharia insurance in terms of transparency are well conveyed.

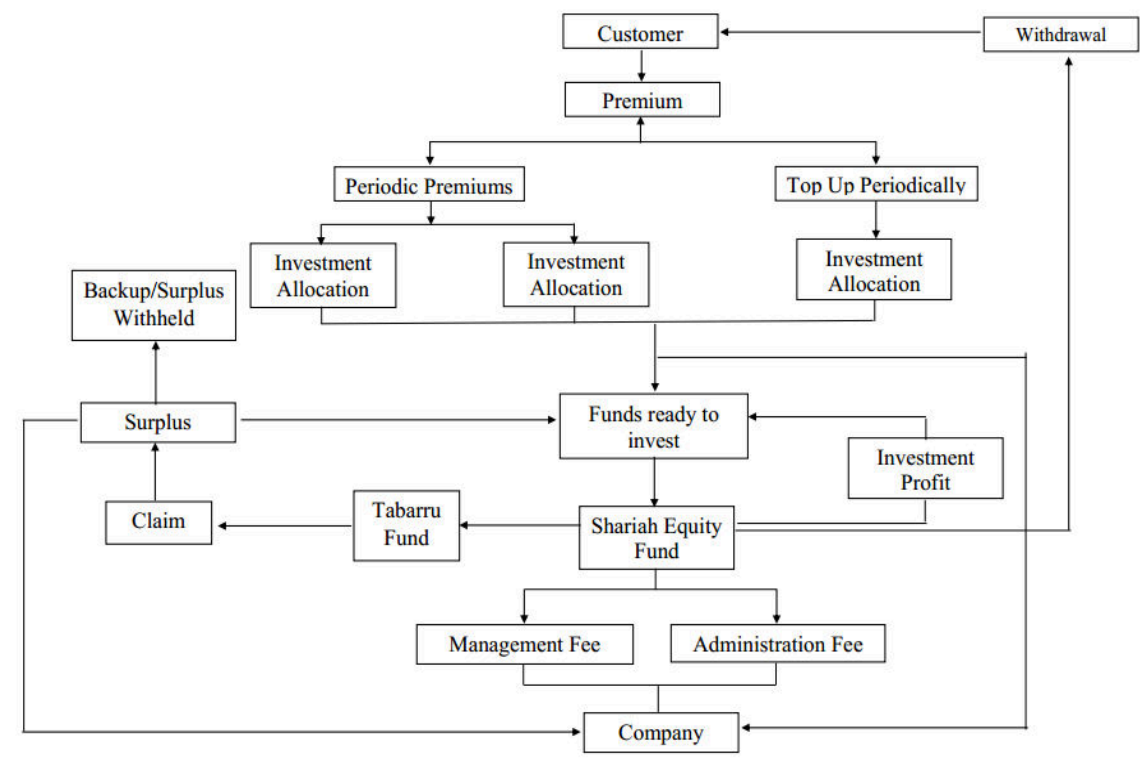

Figure 1. AlliSya Protection Plus Premium Fund Management Scheme

Figure 1 above explains the premium fund management scheme of AlliSya Protection Plus, in which the premiums paid by the customer to the company will be divided into periodic premium accounts (protection) and periodic top up accounts (investment). Periodic premiums will be re-divided into investment portions and acquisition costs. While the periodic top up will all be used as an investment portion. Each investment portion that if combined will be used as an investment fund. In this scheme, the investment instruments taken is sharia equity fund. Investment returns are used for management fees, administrative fees and tabarru funds. Furthermore, tabarru funds will be used for claim payments. If there is a surplus (residual), it will return to being an investment and reserve fund.

Some terms are written on the AlliSya Protection Plus premium fund management scheme, including: [20]

1 Premiums : The amount of money that must be paid by the customer as an obligation of the insured for his participation in insurance

2 Periodic Premiums : Premium portion for predictions 
3 Periodic Top Up : Portion of premium for investment

4 Acquisition Cost : The cost of managing or operating costs to run the business of an insurance company

5 Syariah Equity : An investment fund that aims to obtain optimal investment Fund returns in the medium and long term through the placement of funds in quality stocks in accordance with sharia principles

6 Tabarru : The welfare fund that has been intended by the customer for the purpose of helping and assisting each other, is payable if a customer dies and or the agreement has expired.

7 Claim : An official request to an insurance company, to request payment under the terms of the agreement

8 Surplus : The difference is more/less than the total premium of the customer to the tabarru fund after deducting the payment of compensation/claims, reinsurance contributions, and technical reserves, in a given period.

\section{Ilustration of AlliSya Protection Plus}

Suppose a 37-year-old father insures his 1-year-old daughter with a premium of Rp. 5,000,000 annually, the benefits to be obtained include: [20]

1. The Basic benefits of death compensation in the amount of Rp. 125,000,000 is valid until the protection period is completed.

2. Additional benefits:

a. CI Plus (49 critical illnesses) of Rp. 250,000,000 is valid until the protection period is complete.

b. Allisya Hospital \& Surgical Care of (hospital fees) with Plan 500 (hospital type) is valid until the insured is 80 years old.

c. ADDB Syariah (died or disability due to an accident) of Rp. 100,000,000 applies until the insured is before 65 years old.

d. Payor B. Basic (exemption from paying regular premiums) of Rp. 3,000,000 per year applies until the premium payer is 65 years old.

e. Payor B. TUPR (periodic Top Up payment exemption) of Rp. 2,000,000 per year is valid until the premium payer is 65 years old.

Table 1. The Benefits of AlliSya Hospital and Surgical Care (Plan 500)

\begin{tabular}{|c|c|c|c|c|}
\hline \multirow{2}{*}{ No } & \multirow{2}{*}{ Compensation } & \multirow{2}{*}{ Maximum Limit } & Plan 500 & \multirow{2}{*}{ Description } \\
\hline & & & IDR & \\
\hline 1 & Room & Max 180 days per year & 500.000 & Per day \\
\hline 2 & ICU Room & Max 30 days per year & 700.000 & Per day \\
\hline 3 & $\begin{array}{l}\text { Visit of the Treating } \\
\text { Doctor }\end{array}$ & Max 180 days per year & 150.000 & Per day \\
\hline 4 & Specialist Consultation & Max 180 days per year & 175.000 & Per day \\
\hline 5 & Surgery & & & \\
\hline
\end{tabular}




\begin{tabular}{|c|c|c|c|c|}
\hline & Complex Surgery & Per period of hospitalization & 82.000 .000 & \multirow{9}{*}{$\begin{array}{l}1 \\
\text { hospitalization } \\
\text { period }=30 \\
\text { days }\end{array}$} \\
\hline & Major Surgery & Per period of hospitalization & 53.300 .000 & \\
\hline & Moderate Surgery & Per period of hospitalization & 36.900 .000 & \\
\hline & Small Surgery & Per period of hospitalization & 20.500 .000 & \\
\hline 6 & Miscellaneous charges & Per period of hospitalization & 8.500 .000 & \\
\hline 7 & $\begin{array}{l}\text { Before and After- } \\
\text { admission fee }\end{array}$ & $\begin{array}{l}\text { Per hospitalization period } 30 \\
\text { days before and } 90 \text { days } \\
\text { after }\end{array}$ & 2.000 .000 & \\
\hline 8 & Private Nurse at Home & Max 180 days per year & 250.000 & \\
\hline 9 & $\begin{array}{l}\text { Outpatient and } \\
\text { Emergency Teeth due to } \\
\text { Accidents }\end{array}$ & Per incident for 14 days & 5.000 .000 & \\
\hline 10 & Ambulance & Per period of hospitalization & 350.000 & \\
\hline
\end{tabular}

Complete data of the AlliSya Protection Plus can be viewed through:

https://rive.google.com/file/d/1M35A4y3A1ToFA4u2lxJKdqYr79gos5M4/view?usp=sharing

\section{Mathematical Formulation of AlliSya Protection Plus Integration Model}

AlliSya Protection Plus is a unit link sharia product with a protection period until the insured aged 100 years and premium payments until the insured aged 99 years. The first stage done before compiling the integration model table for AlliSya Protection Plus is to create a proposal table. This table is based on information from the illustrations of AlliSya Protection Plus. This proposal must be clear and cover all possibilities that will occur within the period of insurance contract and are generally referred to as client proposals [5].

Table 2. AlliSya Protection Plus Client Proposal

\begin{tabular}{|c|c|c|c|}
\hline No & Item & Notation & Description \\
\hline 1 & Premium & $\mathrm{P}$ & Rp. 5.000.000 per year \\
\hline 2 & Child: Female non-smokers & $\mathrm{k}$ years & 1 year old \\
\hline 3 & Father & k years & 37 years old \\
\hline 4 & Contract Period & $\mathrm{n}$ years & 100 years old \\
\hline 5 & Periodic Premiums & $\mathrm{Pb}$ & Rp. 3.000.000 per year \\
\hline 6 & Periodic Top Up & Tub & Rp. 2.000.000 per year \\
\hline 7 & Acquisition Cost & W & $75 \%, 40 \%, 15 \%, 7.5 \%, 7.5 \%$ \\
\hline 8 & Investment Allocation & I & $\begin{array}{l}25 \%, 60 \%, 85 \%, 92.5 \%, 92.5 \%, \ldots \\
, 105,26 \%\end{array}$ \\
\hline 9 & Administration Fee & Adm & Rp. 318.000 per year \\
\hline 10 & Management Fee & $\mathrm{Bp}$ & $2 \%$ \\
\hline 11 & Margin (i) & $\mathrm{r}$ (per year) & $5 \%$ \\
\hline 12 & Surrender Value & $\mathrm{Sv}$ & \\
\hline 13 & Tabarru Basic Insurance & $t_{1}$ & Rp. 121.248 \\
\hline
\end{tabular}




\begin{tabular}{llll}
\hline 14 & Tabarru CI Plus & $t_{2}$ & Rp. 227.496 \\
15 & Tabarru Payor B. Basic & $t_{3}$ & Rp. 102.000 \\
16 & Tabarru Payor B. TUPR & $t_{4}$ & Rp. 68.004 \\
17 & Tabarru ADDB Sharia & $t_{5}$ & Rp. 135.000 \\
18 & Tabarru Allisya Hospital \& Surgical & $t_{6}$ & Rp. 2.068 .800 \\
19 & Care & & Rp. 125.000 .000 \\
20 & Casic Insurance & & Rp. 250.000.000 \\
21 & Payor B. Basic & Rp. 3.000.000 per year \\
22 & Payor B. TUPR & Rp. 2.000.000 per year \\
23 & ADDB Sharia & & Rp. 100.000 .000 \\
24 & Allisya Hospital \& Surgical Care + & & Plan 500 \\
\hline
\end{tabular}

Using information from Tables 1 and 2, the mathematical equations needed to compile the AlliSya Protection Plus integration model table include:

a. Premi (P)

$$
\begin{aligned}
& P_{1}=5.000 .000 * 1=5.000 .000 \\
& P_{2}=5.000 .000 * 2=10.000 .000 \\
& P_{3}=5.000 .000 * 3=15.000 .000 \\
& \vdots \\
& P_{n}=P * \mathrm{n}
\end{aligned}
$$

with $n=1,2, \ldots, 99$.

The next premium payments will be divided into 2 accounts, which is $40 \%$ for Periodic Top Up (TUb) and 60\% for Periodic Premiums ( $\mathrm{Pb})$.

b. Periodic Top Up (TUb)

$$
\begin{aligned}
& \mathrm{TUb}_{1}=40 \% * 5.000 .000=2.000 .000 \\
& \mathrm{TUb}_{2}=40 \% * 10.000 .000=4.000 .000 \\
& \mathrm{TUb}_{3}=40 \% * 15.000 .000=6.000 .000 \\
& \vdots \\
& \mathrm{TUb}_{\mathrm{n}}=40 \% * P_{n} \\
& \text { with } n=1,2, \ldots, 99 .
\end{aligned}
$$

c. Periodic Premiums $(\mathrm{Pb})$

$$
\begin{aligned}
& \mathrm{Pb}_{1}=60 \% * 5.000 .000=3.000 .000 \\
& \mathrm{~Pb}_{2}=60 \% * 10.000 .000=6.000 .000 \\
& \mathrm{~Pb}_{3}=60 \% * 15.000 .000=9.000 .000 \\
& \vdots \\
& \mathrm{Pb}_{\mathrm{n}}=60 \% * P_{n} \\
& \text { with } n=1,2, \ldots, 99 .
\end{aligned}
$$

Periodic Top Up accounts are fully invested, while the Periodic Premium accounts for the first 5 years are divided into 2 accounts for acquisition and investment costs, while the 6th year and so on the Periodic Premiums is fully invested and added $5.26 \%$ of Periodic Premiums.

d. Acquisition Cost (W)

$$
\begin{aligned}
& \mathrm{W}_{1}=75 \% \times 3.000 .000=2.250 .000 \\
& \mathrm{~W}_{2}=40 \% \times 3.000 .000=1.200 .000 \\
& \mathrm{~W}_{3}=15 \% \times 3.000 .000=450.000
\end{aligned}
$$


$\mathrm{W}_{4}=7,5 \% \times 3.000 .000=225.000$

$\mathrm{W}_{5}=7,5 \% \times 3.000 .000=225.000$

$\mathrm{W}_{n}=p \% \times \mathrm{Pb}_{1}$

with $n=1, \ldots, 5$.

e. Investment Allocation (I)

$\mathrm{I}_{1}=25 \% \times 3.000 .000=750.000$

$\mathrm{I}_{2}=60 \% \times 3.000 .000=1.800 .000$

$\mathrm{I}_{3}=85 \% \times 3.000 .000=2.550 .000$

$\mathrm{I}_{4}=92,5 \% \times 3.000 .000=2.775 .000$

$I_{5}=92,5 \% \times 3.000 .000=2.775 .000$

$\mathrm{I}_{6}=105,26 \% \times 3.000 .000=3.157 .800$

$\vdots$

$\mathrm{I}_{\mathrm{n}}=q \% * \mathrm{~Pb}_{1}$

with $n=1,2, \ldots, 99$.

f. Investment Funds (DI)

Calculate the investment funds, namely surrender value plus Periodic Top Up plus investment portion.

$\mathrm{DI}_{1}=0+2.000 .000+750.000=2.750 .000$

$\mathrm{DI}_{2}=2.743 .125+2.000 .000+1.800 .000=6.543 .125$

$\mathrm{DI}_{3}=3.550 .450+2.000 .000+2.550 .000=8.100 .450$

:

$\mathrm{DI}_{\mathrm{n}}=\mathrm{Sv}_{\mathrm{n}}+\mathrm{TUb}_{1}+\mathrm{I}_{\mathrm{n}}$

with $n=1,2, \ldots, 100$.

g. Profit $(\mathrm{Pr})$

Calculate the profit which is $5 \%$ of investment funds

$\operatorname{Pr}_{1}=5 \% * 2.750 .000=137.500$

$\operatorname{Pr}_{2}=5 \% * 6.543 .125=327.156$

$\operatorname{Pr}_{3}=5 \% * 8.100 .450=405.022$

:

$\operatorname{Pr}_{\mathrm{n}}=5 \% * \mathrm{DI}_{\mathrm{n}}$

with $n=1,2, \ldots, 100$.

h. Investment Value (NI)

Calculating the value of investment is the sum of investment funds and profits.

$\mathrm{NI}_{1}=2.750 .000+137.500=2.887 .500$

$\mathrm{NI}_{2}=6.543 .125+327.156=6.870 .281$

$\mathrm{NI}_{3}=8.100 .450+405.022=8.505 .4772$

!

$\mathrm{NI}_{\mathrm{n}}=\mathrm{DI}_{\mathrm{n}} * \operatorname{Pr}_{\mathrm{n}}$

with $n=1,2, \ldots, 100$.

i. Management Fee (Bp)

Calculating management costs is $2 \%$ of the investment value

$\mathrm{Bp}_{1}=0$

$\mathrm{Bp}_{2}=0$

$\mathrm{Bp}_{3}=2 \% * 6.870 .281=137.406$

$\vdots$

$\mathrm{Bp}_{\mathrm{n}}=2 \% * \mathrm{NI}_{\mathrm{n}}$

with $n=1,2, \ldots, 100$. 
j. $\quad$ Total Tabarru (T)

Total tabarru is the sum of all tabarru of each benefit

For example:

$t_{1}=$ Basic insurance $=121.248$

$t_{2}=$ CI Plus $=227.496$

$t_{3}=$ Payor B. Basic $=102.000$

$t_{4}=$ Payor B. TUPR $=121.248$

$t_{5}=$ ADDB Syariah $=68.004$

$t_{6}=$ Allisya Hospital \& Surgical Care $=2.068 .800$

Then:

$\mathrm{T}_{1}=121.248+227.496+102.000+121.248+68.004+2.068 .800=2.722 .548$

!

$\mathrm{T}_{\mathrm{n}}=t_{1_{n}}+t_{2 n}+t_{3 n}+t_{4_{n}}+t_{5_{n}}+t_{6_{n}}$

k. The Remaining Balance (S)

$\mathrm{S}_{1}=0$

$\mathrm{S}_{2}=2.887 .500-0-0-0=2.887 .500$

$\mathrm{S}_{3}=6.870 .281-137.406-2.722 .548-318.000=3.692 .328$

$\vdots$

$\mathrm{S}_{\mathrm{n}}=\mathrm{NI}_{\mathrm{n}-1}-\mathrm{BP}_{\mathrm{n}}-\mathrm{T}_{\mathrm{n}}-\mathrm{Adm}$

with $n=1,2, \ldots, 100$.

1. Surrender Value (Sv)

Surrender value is the remaining balance multiplied by $95 \%$, because it assumes $5 \%$ for the difference between buying and selling shares. $\mathrm{Sv}_{1}=0$

$\mathrm{Sv}_{2}=2.887 .500 * 95 \%=2.743 .125$

$\mathrm{Sv}_{3}=3.692 .328 * 95 \%=3.507 .711$

$\vdots$

$\mathrm{Sv}_{\mathrm{n}}=\mathrm{S}_{\mathrm{n}} * 95 \%$

with $n=1,2, \ldots, 100$.

Furthermore from the mathematical equations acquired, will be compiled into Table 3 and named the mathematical equations of AlliSya Protection Plus Integration Model [3], [ 4], [612], [14-17]. 


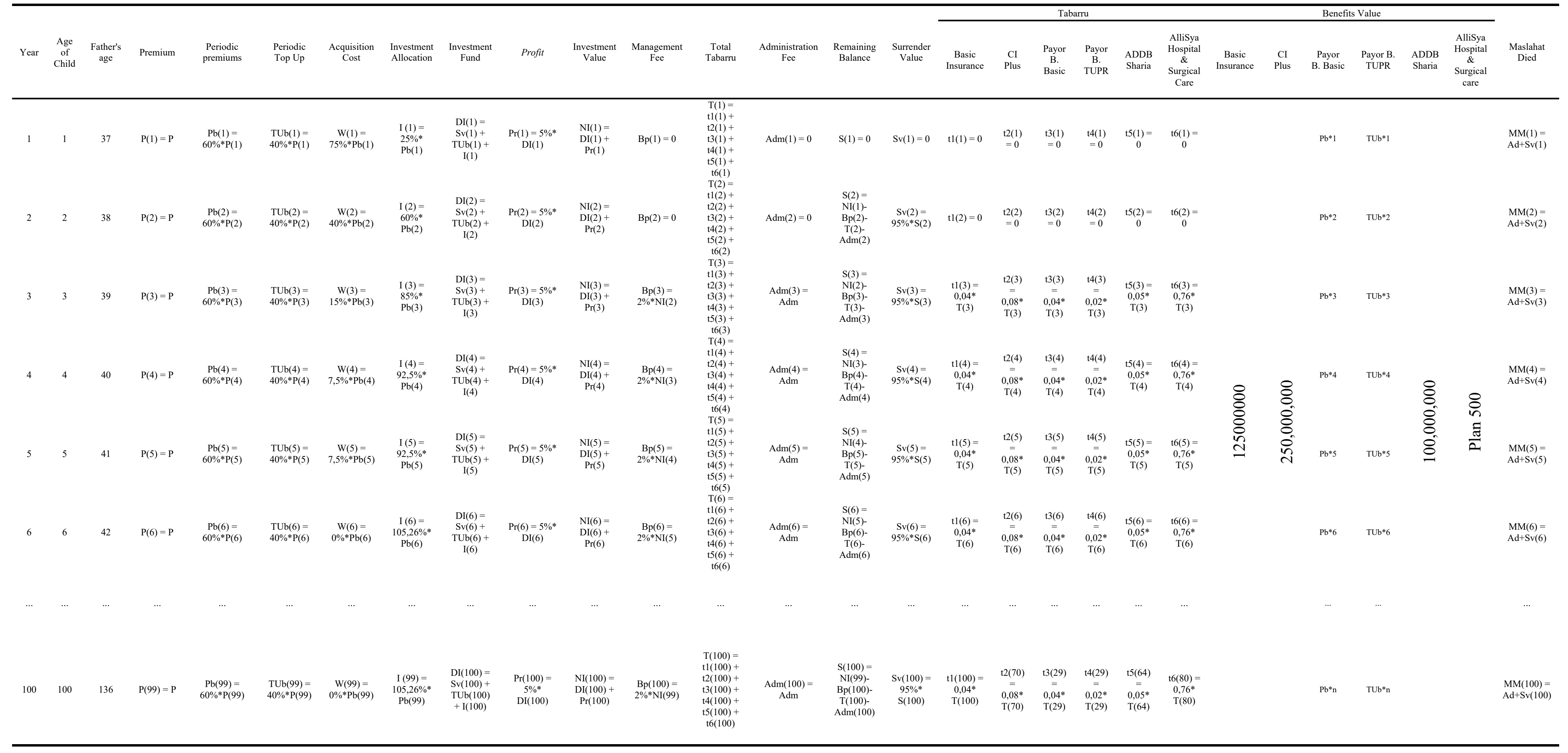

Furthermore, if the premium value of Rp. 5,000,000 per year is substituted into Table 3, then the table of the AlliSya Protection Plus Integration Model is obtained as shown in Table 4 below. 
Table 4. AlliSya Protection Plus Integration Model

\begin{tabular}{|c|c|c|c|c|c|c|c|c|c|c|c|c|c|c|c|c|c|c|c|c|c|c|c|c|c|c|c|c|}
\hline \multirow[b]{2}{*}{ Year } & \multirow[b]{2}{*}{$\begin{array}{l}\text { Age of } \\
\text { Child }\end{array}$} & \multirow[b]{2}{*}{$\begin{array}{c}\text { Fathe } \\
\text { r's } \\
\text { age }\end{array}$} & \multirow[b]{2}{*}{ Premium } & & & & & & & & & & & & & & & & baru & & & & & & its Value & & & \\
\hline & & & & $\begin{array}{c}\text { Periodic } \\
\text { premiums }\end{array}$ & $\begin{array}{l}\text { Periodic } \\
\text { Top Up }\end{array}$ & $\begin{array}{c}\text { Acquisition } \\
\text { Cost }\end{array}$ & $\begin{array}{l}\text { Investment } \\
\text { Allocation }\end{array}$ & $\begin{array}{l}\text { Investment } \\
\text { Fund }\end{array}$ & Profit & $\begin{array}{l}\text { Investment } \\
\text { Value }\end{array}$ & $\begin{array}{l}\text { Managemen } \\
\text { tFee }\end{array}$ & $\begin{array}{c}\text { Total } \\
\text { Tabarru }\end{array}$ & $\begin{array}{l}\text { Administratait } \\
\text { on Fee }\end{array}$ & $\begin{array}{c}\text { Remaining } \\
\text { Balance }\end{array}$ & $\begin{array}{c}\text { Surrender } \\
\text { Value }\end{array}$ & $\begin{array}{c}\text { Basic } \\
\text { Insurance }\end{array}$ & CI Plus & $\begin{array}{c}\begin{array}{c}\text { Payor r. } \\
\text { Basic }\end{array} \\
\text { a }\end{array}$ & $\begin{array}{c}\text { Payor } \\
\text { B } \\
\text { TUPR } \\
\end{array}$ & $\begin{array}{l}\text { ADDB } \\
\text { Sharia }\end{array}$ & $\begin{array}{c}\text { Allisyan } \\
\text { Hospital } \\
\text { Surgical } \\
\text { Care } \\
\text { Care }\end{array}$ & $\begin{array}{c}\text { Basic } \\
\text { Insuran } \\
\text { ce }\end{array}$ & CI Plus & $\begin{array}{c}\text { Payor B. } \\
\text { Basic }\end{array}$ & $\begin{array}{c}\text { Payor B. } \\
\text { TuPR }\end{array}$ & $\begin{array}{c}\text { ADDB } \\
\text { Syariah }\end{array}$ & 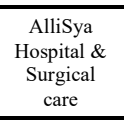 & $\begin{array}{c}\text { Masalahat } \\
\text { Died }\end{array}$ \\
\hline $\mathrm{n}$ & $\mathrm{k}$ & $\mathrm{k}^{\prime}$ & $\mathrm{P}(\mathrm{n})$ & $\begin{array}{c}\mathrm{Pb}(\mathrm{n})= \\
60 \% \text { * } \mathrm{P}(\mathrm{n})\end{array}$ & 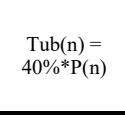 & $\begin{array}{l}\text { W(n) } \\
p \% \%+F b\end{array}$ & $\begin{array}{l}I(n)= \\
p^{2} \sigma^{*} \times \mathrm{Pb}\end{array}$ & $\begin{array}{c}\mathrm{DI}(\mathrm{n})= \\
\operatorname{Tub+1(\mathrm {n})}\end{array}$ & 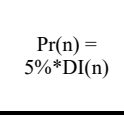 & $\begin{array}{c}\mathrm{NI}(\mathrm{n})= \\
\mathrm{DI}(\mathrm{n}) \operatorname{Pr}(\mathrm{n})\end{array}$ & $\begin{array}{c}B p(n)= \\
2 \% \sigma^{N} \mathrm{~N}(\mathrm{n}-1)\end{array}$ & 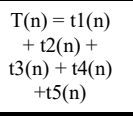 & $\operatorname{Adm}(n)=A d m$ & 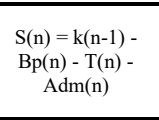 & 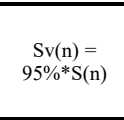 & $\begin{array}{c}\mathrm{t} 1(\mathrm{n})= \\
0,04^{*} \\
\mathrm{~T}(\mathrm{n}) \\
\end{array}$ & $\begin{array}{c}t(n)= \\
0,08^{*} \\
T(n)\end{array}$ & $\begin{array}{c}\mathrm{T}(\mathrm{n})= \\
0,04^{*} \\
\mathrm{~T}(\mathrm{n}) \\
\end{array}$ & $\begin{array}{c}t(\mathrm{n}) \\
= \\
0,02{ }^{*} \\
T(\mathrm{n}) \\
\end{array}$ & $\begin{array}{c}5(3)= \\
0,05 * \\
T(n) \\
\end{array}$ & $\begin{array}{c}\mathrm{t} 6(\mathrm{n})= \\
0,76^{*} \\
\mathrm{~T}(\mathrm{n})\end{array}$ & & & $\mathrm{Pb}^{*} \mathrm{n}$ & $\mathrm{TU}^{*}{ }^{*} \mathrm{n}$ & & & $\begin{array}{l}\mathrm{MM}(\mathrm{n})=\mathrm{Ac} \\
+\mathrm{S}(\mathrm{N})\end{array}$ \\
\hline 1 & 1 & 37 & $\begin{array}{l}5,000,000 \\
\end{array}$ & & $\begin{array}{l}2,000,000 \\
\end{array}$ & $\begin{array}{l}2,250,000 \\
\end{array}$ & $\begin{array}{l}75,0000 \\
\end{array}$ & $\begin{array}{l}2,750,000 \\
\end{array}$ & 137,500 & $2,887,500$ & & & & & & & & & & & & & & $\begin{array}{l}5,000,000 \\
\end{array}$ & $3,000,000$ & & & \\
\hline $\begin{array}{l}2 \\
3\end{array}$ & $\begin{array}{l}2 \\
3\end{array}$ & $\begin{array}{l}38 \\
39\end{array}$ & $\begin{array}{l}10,00,0,000 \\
1,500,000\end{array}$ & $\begin{array}{l}6,000,0000 \\
9,000,000\end{array}$ & $\begin{array}{l}4,000,0000 \\
6,000,000\end{array}$ & $\begin{array}{l}1,200,000 \\
455,000\end{array}$ & $\begin{array}{l}1,800,000 \\
2,550,000\end{array}$ & $\begin{array}{l}6,543,1,25 \\
8,077,711\end{array}$ & $\begin{array}{l}327,156 \\
420.886\end{array}$ & $\begin{array}{l}6,870,281 \\
8,460,597\end{array}$ & 137,406 & $2,722.548$ & 318,000 & $\begin{array}{l}2,887,500 \\
3, ., 692,328\end{array}$ & $\begin{array}{l}2,743,125 \\
3,300,711\end{array}$ & 121,248 & 227,496 & $\begin{array}{l}102.000 \\
\end{array}$ & 68.004 & 135,000 & 2.068 .800 & & & $\begin{array}{l}5,000,000 \\
5,000,000\end{array}$ & $\begin{array}{l}3,000,000 \\
3,00000\end{array}$ & & & $\begin{array}{l}127,743,125 \\
121.807711\end{array}$ \\
\hline 4 & 4 & 40 & $20,000,000$ & $12,000,000$ & $8,000,000$ & 225,000 & $2,775,000$ & $9,763,295$ & 488,165 & $10,251,460$ & 169,212 & $2,722,548$ & 318,000 & $5,250,837$ & $4,988,295$ & 121,248 & 227,496 & 102,000 & $\begin{array}{l}{ }_{0} 0,0,044 \\
68,004\end{array}$ & 135,000 & $2,068,800$ & & & $\begin{array}{l}5,000,000 \\
5,000,000\end{array}$ & $\begin{array}{l}3,000,000 \\
3,0000000\end{array}$ & & & $\begin{array}{l}128,807,711 \\
129,988,295\end{array}$ \\
\hline 5 & 5 & 41 & $25,000,000$ & $15,000,000$ & $10,000,000$ & 225,000 & $2,775,000$ & $11,430,588$ & 57,529 & $12,002,118$ & 205,029 & $2,722,548$ & 318,000 & $7,005,883$ & $6,655,588$ & 121,248 & 227,496 & 102,000 & 68,004 & 135,000 & $2,068,800$ & & & $5,000,000$ & $\begin{array}{l}3,000,000 \\
3\end{array}$ & & & $\begin{array}{l}212,988,2,23 \\
131,655,588\end{array}$ \\
\hline 6 & 6 & 42 & $30,000,000$ & $18,000,000$ & $12,000,000$ & & $3,157,800$ & $13,443,251$ & 672,163 & $14,115,414$ & 240,042 & $2,722,548$ & 318,000 & $8,721,528$ & $8,285,451$ & 121,248 & 227,496 & 102,000 & 68,004 & 135,000 & $2,068,800$ & & & $5,000,000$ & $3,000,000$ & & & $133,285,451$ \\
\hline 7 & 7 & 43 & $35,000,000$ & $21,000,000$ & $14,000,000$ & & $3,157,800$ & $15,410,730$ & 770,536 & $16,181,266$ & 282,308 & $2,722,548$ & 318,000 & $10,792,557$ & $\begin{array}{r}10,252,930 \\
\end{array}$ & 121,248 & 227,496 & 102,000 & 68,004 & 135,000 & $2,068,800$ & & & $5,000,000$ & $3,000,000$ & & & $135,252,930$ \\
\hline 8 & 8 & 44 & $40,000,000$ & $24,000,000$ & $16,000,000$ & - & $3,157,800$ & $17,334,038$ & 866,702 & $18,200,740$ & 323,625 & $2,722,548$ & 318,000 & $12,817,093$ & $\begin{array}{l}12,176,238 \\
\end{array}$ & 121,248 & 227,496 & 102,000 & 68,004 & 135,000 & $2,068,800$ & & & $5,000,000$ & $3,000,000$ & & & $137,17,238$ \\
\hline 9 & 9 & 45 & $45,000,000$ & $27,000,000$ & $18,000,000$ & - & $3,157,800$ & $19,214,168$ & 960,708 & $20,174,877$ & 364,015 & $2,722,548$ & 318,000 & $14,796,177$ & $14,056,368$ & 121,248 & 227,496 & 102,000 & 68,004 & 135,000 & $2,068,800$ & & & $5,000,000$ & $3,000,000$ & & & $139,05,368$ \\
\hline 10 & 10 & 46 & $50,000,000$ & $30,000,000$ & $20,000,000$ & - & $3,157,800$ & $21,052,090$ & $1,052,604$ & $22,104,694$ & 403,498 & $2,722,548$ & 318,000 & $16,730,831$ & $15,894,290$ & 121,248 & 227,496 & 102,000 & 68,004 & 135,000 & $2,068,800$ & & & $5,000,000$ & $3,000,000$ & & & $140,894,290$ \\
\hline 11 & 11 & 47 & $55,000,000$ & $33,000,000$ & $22,000,000$ & - & $3,157,800$ & $22,848,750$ & $1,142,437$ & $23,991,187$ & 442,094 & $2,722,548$ & 318,000 & $18,622,052$ & $\begin{array}{l}17,690,950 \\
\end{array}$ & 121,248 & 227,496 & 102,000 & 68,004 & 135,000 & $2,068,800$ & & & $5,000,000$ & $3,000,000$ & & & $142,69,950$ \\
\hline 12 & 12 & 48 & $60,000,000$ & $36,000,000$ & $24,000,000$ & & $3,157,800$ & $24,605,075$ & $1,230,254$ & $25,835,328$ & 479,824 & $2,722,548$ & 318,000 & $20,470,815$ & $19,447,275$ & 121,248 & 227,496 & 102,000 & 68,004 & 135,000 & $2,068,800$ & & & $5,000,000$ & $3,000,000$ & & & $144,447,275$ \\
\hline 13 & 13 & 49 & $65,000,000$ & $39,000,000$ & $26,000,000$ & & $3,157,800$ & $26,321,970$ & $1,316,099$ & $27,638,069$ & 516,707 & $2,722,548$ & 318,000 & $22,278,074$ & $21,164,170$ & 121,248 & 227,496 & 102,000 & 68,004 & 135,000 & $2,068,800$ & & & $5,000,000$ & $3,000,000$ & & & $146,164,170$ \\
\hline 14 & 14 & 50 & $70,000,000$ & $42,000,000$ & $28,000,000$ & & $3,157,800$ & $28,000,321$ & $1,400,016$ & $29,400,337$ & 552,761 & $2,722,548$ & 318,000 & $24,044,759$ & $22,842,521$ & 121,248 & 227,496 & 102,000 & 68,004 & 135,000 & $2,068,800$ & & & $5,000,000$ & $3,000,000$ & & & $147,842,521$ \\
\hline 15 & 15 & 51 & $75,000,000$ & $45,000,000$ & $30,000,000$ & & $3,157,800$ & $29,640,993$ & $1,482,050$ & $31,123,043$ & 588,007 & $2,722,548$ & 318,000 & $25,771,783$ & $24,483,193$ & 121,248 & 227,496 & 102,000 & 68,004 & 135,000 & $2,068,800$ & & & $5,000,000$ & $3,000,000$ & & & $149,48,193$ \\
\hline 16 & 16 & 52 & $80,000,000$ & $48,000,000$ & $32,000,000$ & - & $3,157,800$ & $31,244,833$ & $1,562,242$ & $32,807,074$ & 622,461 & $2,722,548$ & 318,000 & $27,460,034$ & $26,087,033$ & 121,248 & 227,496 & 102,000 & 68,004 & 135,000 & $2,068,800$ & & & $5,000,000$ & $3,000,000$ & & & $151,087,033$ \\
\hline 17 & 17 & 53 & $85,000,000$ & $51,000,000$ & $34,000,000$ & - & $3,157,800$ & $32,812,665$ & $1,640,633$ & $34,453,299$ & 65,141 & $2,722,548$ & 318,000 & $29,110,385$ & $27,654,865$ & 121,248 & 227,496 & 102,000 & 68,004 & 135,000 & $2,066,800$ & & & $5,000,000$ & $3,000,000$ & & & $152,64,865$ \\
\hline 18 & 18 & 54 & $90,000,000$ & $54,000,000$ & $36,000,000$ & - & $3,157,800$ & $34,345,301$ & $1,717,265$ & $36,062,566$ & 689,066 & $2,722,548$ & 318,000 & $30,723,685$ & $29,187,501$ & 121,248 & 227,496 & 102,000 & 68,004 & 135,000 & $2,068,800$ & & & $5,000,000$ & $3,000,000$ & & & $154,187,501$ \\
\hline 19 & 19 & 55 & $95,000,000$ & $57,000,000$ & $38,000,000$ & - & $3,157,800$ & $35,843,528$ & $1,792,176$ & $37,635,704$ & 721,251 & $2,722,548$ & 318,000 & $32,300,766$ & $30,685,728$ & 121,248 & 227,496 & 102,000 & 68,004 & 135,000 & $2,068,800$ & & & $5,000,000$ & $3,000,000$ & & & $155,685,728$ \\
\hline 20 & 20 & 56 & $100,000,000$ & 60,000,000 & $40,000,000$ & - & $3,157,800$ & $37,308,120$ & $\begin{array}{r}1,865,406 \\
, 0,900\end{array}$ & $39,173,526$ & 752,714 & $2,722,548$ & 318,000 & $33,842,442$ & $32,150,320$ & 121,248 & 227,446 & 102,000 & 68,004 & 135,000 & $2,068,800$ & & & $5,000,000$ & $3,000,000$ & & & $157,150,320$ \\
\hline 21 & 21 & 57 & $105,000,000$ & $63,000,000$ & $42,000,000$ & - & $\begin{array}{l}3,157,800 \\
, 1,5600\end{array}$ & $38,739,832$ & $\begin{array}{l}1,936,992 \\
\end{array}$ & $\begin{array}{l}40,676,824 \\
21,6,20\end{array}$ & $\begin{array}{l}783,471 \\
0.9352\end{array}$ & $\begin{array}{l}2,722,548 \\
, 7205\end{array}$ & 318,000 & $\begin{array}{l}35,349,508 \\
, 6027\end{array}$ & $\begin{array}{r}33,582,032 \\
3,20102\end{array}$ & 121,248 & $\begin{array}{r}227,496 \\
27706\end{array}$ & $\begin{array}{l}102,000 \\
\end{array}$ & 68,004 & 135,000 & $2,068,800$ & & & $5,000,000$ & $3,000,000$ & & & $158,58,032$ \\
\hline 22 & 22 & 58 & $\begin{array}{l}110,000,000 \\
15\end{array}$ & $66,000,000$ & $44,000,000$ & & $\begin{array}{l}3,157,800 \\
3,1,5000\end{array}$ & $\begin{array}{l}40,139,402 \\
, 557,50\end{array}$ & $\begin{array}{l}2,006,970 \\
, 205678\end{array}$ & $\begin{array}{l}42,146,372 \\
\end{array}$ & 813,536 & $\begin{array}{l}2,722,548 \\
, 2725\end{array}$ & 318,000 & $\begin{array}{l}36,822,739 \\
382,089\end{array}$ & $\begin{array}{r}34,981,602 \\
3,620502\end{array}$ & 121,248 & $\begin{array}{l}227,496 \\
2706\end{array}$ & $\begin{array}{l}102,000 \\
\end{array}$ & 68,004 & 135,000 & $2,068,800$ & & & $5,000,000$ & $3,000,000$ & & & $159,981,602$ \\
\hline${ }^{23}$ & 23 & $\begin{array}{l}59 \\
60\end{array}$ & $\begin{array}{l}115,000,000 \\
1\end{array}$ & $\begin{array}{l}69,000,000 \\
72000000\end{array}$ & $\begin{array}{r}46,000,0000 \\
48,000000\end{array}$ & & $\begin{array}{l}3,157,800 \\
3,1,75000\end{array}$ & $\begin{array}{r}41,507,552 \\
\end{array}$ & $\begin{array}{l}2,075,378 \\
, 1,272,0\end{array}$ & $\begin{array}{l}43,582,930 \\
4,007\end{array}$ & $\begin{array}{l}842,927 \\
871650\end{array}$ & $\begin{array}{l}2,722,548 \\
2727564\end{array}$ & 318,000 & $\begin{array}{l}38,262,897 \\
3067772\end{array}$ & $\begin{array}{r}36,349,752 \\
37607197\end{array}$ & 121,248 & 227,496 & 102,000 & 68,004 & 135,000 & $2,068,800$ & & & $5,000,000$ & $3,000,000$ & & & $161,349,752$ \\
\hline${ }^{24}$ & 24 & $\begin{array}{l}60 \\
61\end{array}$ & $\begin{array}{l}120,000,000 \\
125000000\end{array}$ & $\begin{array}{l}72,000,000 \\
75,000000\end{array}$ & $\begin{array}{l}48,000,000 \\
50,00.0000\end{array}$ & - & $\begin{array}{l}3,157,800 \\
3.58700\end{array}$ & $\begin{array}{l}42,844,987 \\
44,152,296\end{array}$ & $\begin{array}{l}2,142,249 \\
2027620\end{array}$ & $\begin{array}{l}44,987,236 \\
463000\end{array}$ & $\begin{array}{l}871,659 \\
800741\end{array}$ & $\begin{array}{l}2,722,548 \\
27205545\end{array}$ & $\begin{array}{l}318,000 \\
318000\end{array}$ & $\begin{array}{l}39,670,723 \\
1406904\end{array}$ & $37,687,187$ & 121,248 & $\begin{array}{l}227,496 \\
2706\end{array}$ & 102,000 & 68,004 & 135,000 & $\begin{array}{l}2,068,800 \\
0,0000\end{array}$ & & & $5,000,000$ & $3,000,000$ & & & $162,687,187$ \\
\hline $\begin{array}{l}25 \\
26\end{array}$ & $\begin{array}{l}25 \\
26\end{array}$ & $\begin{array}{l}61 \\
62\end{array}$ & $\begin{array}{l}125,000,000 \\
130,000,000\end{array}$ & $\begin{array}{l}17,0000,000 \\
78,000,000\end{array}$ & $\begin{array}{l}50,0,0,0,000 \\
52,000,000\end{array}$ & $\therefore$ & $\begin{array}{l}3,1,177,800 \\
3,157,800\end{array}$ & $\begin{array}{l}4,4,152,396 \\
4,530,055\end{array}$ & $\begin{array}{l}2,2027,120 \\
2,27,523\end{array}$ & $\begin{array}{l}46,360,016 \\
47701979\end{array}$ & $\begin{array}{l}899,745 \\
9227200\end{array}$ & $\begin{array}{l}2,722,548 \\
2,722548\end{array}$ & $\begin{array}{l}318,000 \\
318,000\end{array}$ & $\begin{array}{l}41,046,944 \\
42,322688\end{array}$ & $\begin{array}{l}38,994,596 \\
40,272,555\end{array}$ & $\begin{array}{l}121,248 \\
101248\end{array}$ & $\begin{array}{l}227,496 \\
272706\end{array}$ & $\begin{array}{l}102,000 \\
10000\end{array}$ & 68,004 & $\begin{array}{l}135,000 \\
1355000\end{array}$ & & & & $5,000,000$ & $\begin{array}{l}3,000,000 \\
3,00000\end{array}$ & & & $\begin{array}{l}163,994,596 \\
16572,659\end{array}$ \\
\hline 27 & 27 & 63 & $135,000,000$ & $81,000,000$ & $54,000,000$ & . & $3,157,800$ & $46,679,822$ & $2,333,991$ & $\begin{array}{l}4,, 10,117,711 \\
49,0131\end{array}$ & $\begin{array}{l}92,2,200 \\
954,040\end{array}$ & $\begin{array}{l}2,722,548 \\
2,722,548\end{array}$ & $\begin{array}{l}318,000 \\
318,000\end{array}$ & $\begin{array}{l}42,332,268 \\
4,707,390\end{array}$ & $\begin{array}{l}\begin{array}{r}40,27,2,555 \\
4,1,25,2020\end{array} \\
4\end{array}$ & $\begin{array}{l}121,248 \\
121,248\end{array}$ & $\begin{array}{l}227,496 \\
227966\end{array}$ & $\begin{array}{l}102,000 \\
{ }_{10}^{1020000}\end{array}$ & $\begin{array}{l}68,004 \\
68.04\end{array}$ & $\begin{array}{l}133,5000 \\
13,5000\end{array}$ & $\begin{array}{l}2,066,800 \\
20608800\end{array}$ & & & $\begin{array}{l}5,000,000 \\
5,000,000\end{array}$ & $\begin{array}{l}3,000,000 \\
3,0000000\end{array}$ & & & $\begin{array}{l}165,272,655 \\
165920202\end{array}$ \\
\hline 28 & 28 & 64 & $140,000,000$ & $84,000,000$ & $56,000,000$ & & $3,157,800$ & $47,901,138$ & $2,395,057$ & $50,296,195$ & 980,276 & $2,722,548$ & 318,000 & $44,992,987$ & $42,743,338$ & $\begin{array}{l}121,240 \\
121,248\end{array}$ & $\begin{array}{l}227,400 \\
227,496\end{array}$ & 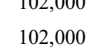 & $\begin{array}{l}68,004 \\
68,004\end{array}$ & 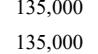 & $\begin{array}{l}2,206,8,800 \\
2,068,800\end{array}$ & ○ & 요 & $\begin{array}{l}5,000,000 \\
5,000,000\end{array}$ & $\begin{array}{l}3,000,000 \\
3,000,000 \\
3\end{array}$ & 8 & & $\begin{array}{l}16,6,522,020 \\
167,743,38\end{array}$ \\
\hline 29 & 29 & 65 & $145,000,000$ & $87,000,000$ & $58,000,000$ & & $3,157,800$ & $49,095,037$ & $2,454,752$ & $51,549,788$ & $1,005,924$ & $2,722,548$ & 318,000 & $46,249,723$ & $43,937,237$ & 121,248 & 227,496 & 102,000 & 68,004 & 135,000 & $2,068,800$ & 8 & & $5,000,000$ & $3,000,000$ & $c$ & 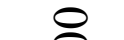 & $168,937,237$ \\
\hline 30 & 30 & 66 & $150,000,000$ & $90,000,000$ & $60,000,000$ & - & $3,157,800$ & $50,423,366$ & $2,521,182$ & $52,944,818$ & $1,033,996$ & $2,552,544$ & 318,000 & $\begin{array}{l}47,648,249 \\
\end{array}$ & $45,265,836$ & 121,248 & 227,496 & & & 135,000 & $2,068,800$ & 0 & $c$ & & & & $D$ & $170,265,836$ \\
\hline 31 & 31 & 67 & $155,000,000$ & $93,000,000$ & $62,000,000$ & - & $3,157,800$ & $51,722,409$ & $2,586,120$ & $54,308,529$ & $1,058,896$ & $2,552,544$ & 318,000 & $49,015,378$ & $46,564,609$ & 121,248 & 227,496 & & & 135,000 & $2,068,800$ & ○ & & & & & 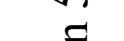 & $171,564,609$ \\
\hline 32 & 32 & 68 & $160,000,000$ & $96,000,000$ & $64,000,000$ & - & $3,157,800$ & $52,992,024$ & $2,649,601$ & $55,641,625$ & $1,086,171$ & $2,552,544$ & 318,000 & $50,351,815$ & $47,834,224$ & 121,248 & 227,496 & & & 135,000 & $2,068,800$ & 0. & & & & 0 & $\bar{\epsilon}$ & $172,834,224$ \\
\hline 33 & 33 & 69 & $165,000,000$ & $99,000,000$ & $66,000,000$ & - & $3,157,800$ & $54,233,136$ & $2,711,657$ & $56,944,793$ & $1,112,833$ & $2,552,544$ & 318,000 & $51,658,249$ & $49,075,336$ & 121,248 & 227,496 & & & 135,000 & $2,068,800$ & $\approx$ & 욤 & & & 8 & $\bar{a}$ & $174,075,336$ \\
\hline 34 & ${ }_{34}$ & 70 & $177,000,000$ & 102,000,000 & $68,000,000$ & - & $3,157,800$ & $55,446,385$ & $2,772,319$ & $58,218,705$ & $1,138,896$ & $2,552,544$ & 318,000 & $52,935,353$ & $50,288,585$ & 121,248 & 227,496 & & & 135,000 & $2,068,800$ & 工 & ก & & & $\underline{-}$ & & $\begin{array}{l}175,288,585 \\
\end{array}$ \\
\hline 35 & 35 & 71 & $175,000,000$ & $105,000,000$ & $70,000,000$ & & $3,157,800$ & $56,632,397$ & $2,881,620$ & $59,464,017$ & $1,164,374$ & $2,552,544$ & 318,000 & $54,183,787$ & $51,474,597$ & 121,248 & 227,496 & & & 135,000 & $2,068,800$ & & & & & & & $\begin{array}{l}176,474,597 \\
\end{array}$ \\
\hline 36 & 36 & 72 & $180,000,000$ & $108,000,000$ & $72,000,000$ & & $3,157,800$ & $57,791,783$ & $2,889,589$ & $60,681,372$ & $1,189,280$ & $2,552,544$ & 318,000 & $55,404,193$ & $52,633,983$ & 121,248 & 227,496 & & & 135,000 & $2,068,800$ & & & & & & & $177,633,983$ \\
\hline 37 & 37 & 73 & $185,000,000$ & $111,000,000$ & $74,000,000$ & & $3,157,800$ & $58,925,141$ & 2,944,257 & $61,871,398$ & $1,213,627$ & $2,552,544$ & 318,000 & $56,597,201$ & $53,767,341$ & 121,248 & 227,496 & & & 135,000 & $2,068,800$ & & & & & & & $178,76,341$ \\
\hline 38 & 38 & 74 & $190,000,000$ & $114,000,000$ & $76,000,000$ & & $3,157,800$ & $60,033,055$ & $3,001,653$ & $63,034,707$ & $1,237,428$ & $2,552,544$ & 318,000 & $57,763,426$ & $54,875,255$ & 121,248 & 227,496 & & & 135,000 & $2,068,800$ & & & & & & & $\begin{array}{l}179,877,255 \\
\end{array}$ \\
\hline 39 & 39 & 75 & $195,000,000$ & $117,000,000$ & $78,000,000$ & - & $3,157,800$ & $61,116,096$ & $3,055,805$ & $64,171,901$ & $1,260,694$ & $2,552,544$ & 318,000 & $58,903,469$ & $55,958,296$ & 121,248 & 227,496 & & & 135,000 & $2,068,800$ & & & & & & & $180,958,296$ \\
\hline 40 & 40 & 76 & $200,000,000$ & $120,000,000$ & $80,000,000$ & - & $3,157,800$ & $62,174,823$ & $3,108,741$ & $65,283,564$ & $1,283,438$ & $2,552,544$ & 318,000 & $60,017,919$ & $57,017,023$ & 121,248 & 227,496 & & & 135,000 & $2,068,800$ & & & & & & & $182,017,023$ \\
\hline 41 & 41 & 77 & $205,000,000$ & $123,000,000$ & $82,000,000$ & - & $3,157,800$ & $63,209,781$ & 3,160,489 & $66,370,270$ & $1,305,671$ & $2,552,544$ & 318,000 & $61,107,348$ & $58,01,981$ & 121,248 & 227,496 & & & 135,000 & $2,068,800$ & & & & & & & $183,051,981$ \\
\hline 42 & 42 & 78 & $210,000,000$ & $126,000,000$ & $84,000,000$ & - & $3,157,800$ & $.221,505$ & $3,211,075$ & $67,432,580$ & $1,327,405$ & $2,552,544$ & 31 & $62,172,321$ & $59,063,705$ & 121,248 & 227,496 & & & 135,000 & $2,068,800$ & & & & & & & $184,063,705$ \\
\hline 43 & 43 & 79 & $215,000,000$ & , ,000,000 & $86,000,000$ & - & $3,157,800$ & . 210,515 & $3,260,526$ & $68,471,041$ & $1,348,652$ & $2,552,544$ & & $63,213,384$ & $60,052,715$ & 248 & 227,496 & & & 135,000 & $2,068,800$ & & & & & & & $185,052,715$ \\
\hline 44 & 44 & 80 & $220,000,000$ & $132,000,000$ & $88,000,000$ & & $3,157,800$ & 177,322 & $3,308,866$ & $69,486,188$ & $1,369,421$ & $2,552,544$ & & $64,231,076$ & $61,019,522$ & 248 & 227,496 & & & 135,000 & $2,068,800$ & & & & & & & $186,019,522$ \\
\hline 45 & 45 & 81 & $225,000,000$ & .000,000 & $90,000,000$ & & $3,157,800$ & $.122,425$ & $3,356,121$ & $70,478,546$ & $1,389,724$ & $2,552,544$ & & $65,225,921$ & $61,964,625$ & 1,248 & 227,496 & & & 135,000 & $2,068,800$ & & & & & & & $186,964,625$ \\
\hline 46 & 46 & 82 & $230,000,000$ & 000,000 & $92,000,000$ & - & $3,157,800$ & $68,046,309$ & $3,402,315$ & $71,448,625$ & 1,40 & $2,552,3$ & & $66,198,431$ & $62,888,509$ & 1,248 & 227,496 & & & 135,000 & $2,068,800$ & & & & & & & $187,88,509$ \\
\hline 47 & 47 & 83 & $235,000,000$ & $.000,000$ & $\begin{array}{l}94,000,000 \\
\end{array}$ & - & $3,157,800$ & $68,949,453$ & $3,447,473$ & $72,396,925$ & 1,42 & $2,5$. & & 67,1 & 63,7 & 121,248 & 227,496 & & & 135,000 & $2,068,800$ & & & & & & & $188,791,653$ \\
\hline 48 & 48 & 84 & $240,000,000$ & .000,000 & $96,000,000$ & ${ }^{-}$ & $3,157,800$ & $69,832,321$ & $3,491,616$ & 73,32 & 1,447 & 2,5 & & & $64,674,521$ & 121,248 & 227,496 & & & 133,000 & $2,068,800$ & & & & & & & $188,674,521$ \\
\hline 49 & 49 & 85 & $245,000,000$ & 000 & 100 & - & $3,157,800$ & $70,695,368$ & $3,534,768$ & 74,2 & & & & & 65,3 & 121,248 & 227 & & & & $2,068,800$ & & & & & & & $190,57,568$ \\
\hline 50 & 50 & 86 & $250,000,000$ & 0,000 & $100,000,000$ & & 3,157 & $71,539,041$ & $3,576,952$ & & & & & & & 121,248 & 227 & & & & $2,068,800$ & & & & & & & 1,241 \\
\hline 51 & 51 & 87 & 0,000 & , $, 000,000$ & 10 & & & & 3,61 & & & & & & & & 227 & & & & $2,068,800$ & & & & & & & $2,205,972$ \\
\hline 52 & 52 & 88 & $260,000,000$ & $156,000,000$ & $104,000,000$ & - & $3,157,800$ & 989 & $3,658,499$ & 76,82 & 1,51 & & 318 & & $68,012,189$ & 121,248 & 227,496 & & & 135,000 & $2,068,800$ & & & & & & & $193,012,189$ \\
\hline 53 & 53 & 89 & $265,000,000$ & $159,000,000$ & $106,000,000$ & - & $3,157,800$ & 9058,106 & $\begin{array}{l}3,697,905 \\
, 970\end{array}$ & 77,65 & 1,536 & & 318, & 72,42 & $68,800,306$ & 121,248 & 227,496 & & & 135,000 & $2,068,800$ & & & & & & & $193,800,306$ \\
\hline${ }_{54}^{54}$ & 54 & 90 & $\begin{array}{l}270,000,000 \\
\end{array}$ & $162,000,000$ & $108,00,000$ & - & $3,157,800$ & $\begin{array}{l}74,728,530 \\
\end{array}$ & $\begin{array}{l}3,736,426 \\
, 77,006\end{array}$ & & 1,553 & & 318, & $\begin{array}{l}73,23 \\
7,0\end{array}$ & 69,5 & 121,248 & $\begin{array}{l}227,496 \\
2706\end{array}$ & & & 135,000 & $\begin{array}{l}2,068,800 \\
2,08000\end{array}$ & & & & & & & $\begin{array}{l}.570,730 \\
m 72057\end{array}$ \\
\hline 55 & 55 & $\begin{array}{l}91 \\
92\end{array}$ & $275,000,000$ & $165,000,000$ & $110,000,000$ & - & $3,157,800$ & $\begin{array}{l}75,481,657 \\
\end{array}$ & $\begin{array}{l}3,774,083 \\
\end{array}$ & $79,255,740$ & 1,569, & $\begin{array}{l}2,552,544 \\
2,52\end{array}$ & 318, & $\begin{array}{l}74,02 \\
7,000\end{array}$ & $\begin{array}{l}70,323,857 \\
7,0007\end{array}$ & 121,248 & $\begin{array}{l}227,496 \\
2706\end{array}$ & & & 135,000 & $\begin{array}{l}2,068,800 \\
\end{array}$ & & & & & & & $199,323,857$ \\
\hline $\begin{array}{l}56 \\
57\end{array}$ & $\begin{array}{l}56 \\
57\end{array}$ & $\begin{array}{l}92 \\
93\end{array}$ & $\begin{array}{l}280,000,000 \\
285000000\end{array}$ & $\begin{array}{l}168,000,000 \\
1171000\end{array}$ & $112,000,000$ & . & $\begin{array}{l}3,157,800 \\
2,1,5000\end{array}$ & $, \quad, 217,877$ & $\begin{array}{r}3,810,894 \\
3.6978\end{array}$ & $\begin{array}{l}80,028,771 \\
\end{array}$ & $\begin{array}{l}1,585,115 \\
1,00575\end{array}$ & $\begin{array}{l}2,552,5 \\
2,550,\end{array}$ & 318, & $\begin{array}{l}74,80 \\
7555\end{array}$ & 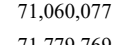 & $\begin{array}{l}121,248 \\
121248\end{array}$ & $\begin{array}{l}227,496 \\
2706\end{array}$ & & & 135,000 & $\begin{array}{l}2,068,800 \\
2,06000\end{array}$ & & & & & & & $19,060,077$ \\
\hline $\begin{array}{l}57 \\
58\end{array}$ & & 93 & $\begin{array}{l}285,000,000 \\
20000\end{array}$ & $\begin{array}{l}171,000,000 \\
1174000\end{array}$ & $\begin{array}{l}114,000,000 \\
16000000\end{array}$ & - & $\begin{array}{l}3,157,800 \\
3,1,77000\end{array}$ & $\begin{array}{c}76,937,569 \\
77,641,19\end{array}$ & $\begin{array}{l}3,846,878 \\
3.892055\end{array}$ & $\begin{array}{l}80,784,448 \\
81523159\end{array}$ & $\begin{array}{l}1,600,575 \\
1616,689\end{array}$ & $\begin{array}{l}2,552,544 \\
2552544\end{array}$ & 318,000 & $\begin{array}{l}75,557,652 \\
762089211\end{array}$ & $\begin{array}{l}71,779,769 \\
7720202\end{array}$ & $\begin{array}{l}121,248 \\
121248\end{array}$ & $\begin{array}{l}227,496 \\
227966\end{array}$ & & & 135,000 & $\begin{array}{l}2,068,800 \\
2,69000\end{array}$ & & & & & & & $\begin{array}{l}196,779,769 \\
107092000\end{array}$ \\
\hline $\begin{array}{l}58 \\
59\end{array}$ & $\begin{array}{l}58 \\
59 \\
59\end{array}$ & $\begin{array}{l}94 \\
95\end{array}$ & $\begin{array}{l}290,00,00,000 \\
29550,00000\end{array}$ & $\begin{array}{l}174,000,000 \\
117000000\end{array}$ & $\begin{array}{l}116,000,000 \\
18180000000\end{array}$ & & $\begin{array}{l}3,157,800 \\
3,1,77000\end{array}$ & $\begin{array}{l}77,641,1,04 \\
78,38.84\end{array}$ & $\begin{array}{l}3,882,055 \\
3.19642\end{array}$ & $\begin{array}{l}81,52,3,159 \\
82,24587\end{array}$ & $\begin{array}{l}1,615,689 \\
1,6304063\end{array}$ & $\begin{array}{l}2,552,544 \\
2,5552544\end{array}$ & $\begin{array}{l}318,000 \\
318,8000\end{array}$ & $\begin{array}{l}76,298,215 \\
77022.15\end{array}$ & $\begin{array}{l}72,48,304 \\
737171044\end{array}$ & $\begin{array}{l}121,248 \\
121248\end{array}$ & $\begin{array}{l}227,496 \\
227966\end{array}$ & & & $\begin{array}{l}133,5000 \\
135000\end{array}$ & $\begin{array}{l}2,068,800 \\
20608000\end{array}$ & & & & & & & , 483,304 \\
\hline 60 & 60 & 96 & $\begin{array}{l}0,000,0,000 \\
0,00,000\end{array}$ & $\begin{array}{l}1177,000,000 \\
180,000,000\end{array}$ & $\begin{array}{l}178,000,0,000 \\
120,000,000\end{array}$ & & 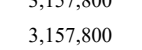 & $\begin{array}{l}78,3,28,844 \\
79,001,145\end{array}$ & $\begin{array}{l}3,9,9,042 \\
3,950,057\end{array}$ & $\begin{array}{l}8,242,5,87 \\
82,951,202\end{array}$ & 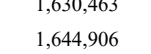 & $\begin{array}{l}2,352,344 \\
2,552,544\end{array}$ & $\begin{array}{l}\begin{array}{r}318,000 \\
318,000\end{array} \\
3\end{array}$ & $\begin{array}{l}77,022,1,122 \\
77,729,837\end{array}$ & $\begin{array}{l}7,1,1,1,4044 \\
73,443,445\end{array}$ & $\begin{array}{l}121,1248 \\
121,248\end{array}$ & $\begin{array}{l}222,7,40 \\
227,496\end{array}$ & & & 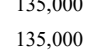 & $\begin{array}{l}2,200,8000 \\
2,068,800\end{array}$ & & & & & & & $\begin{array}{l}198,8,84,34,345 \\
19\end{array}$ \\
\hline 61 & 61 & 97 & $305,000,000$ & $183,000,000$ & $122,000,000$ & & $3,157,800$ & $79,658,352$ & $3,982,918$ & $83,641,270$ & $1,659,024$ & $2,552,544$ & 318,000 & $78,421,634$ & $74,500,552$ & 121,248 & 227,496 & & & 135,000 & $2,068,800$ & & & & & & & $\begin{array}{r}199,500,552 \\
\end{array}$ \\
\hline
\end{tabular}




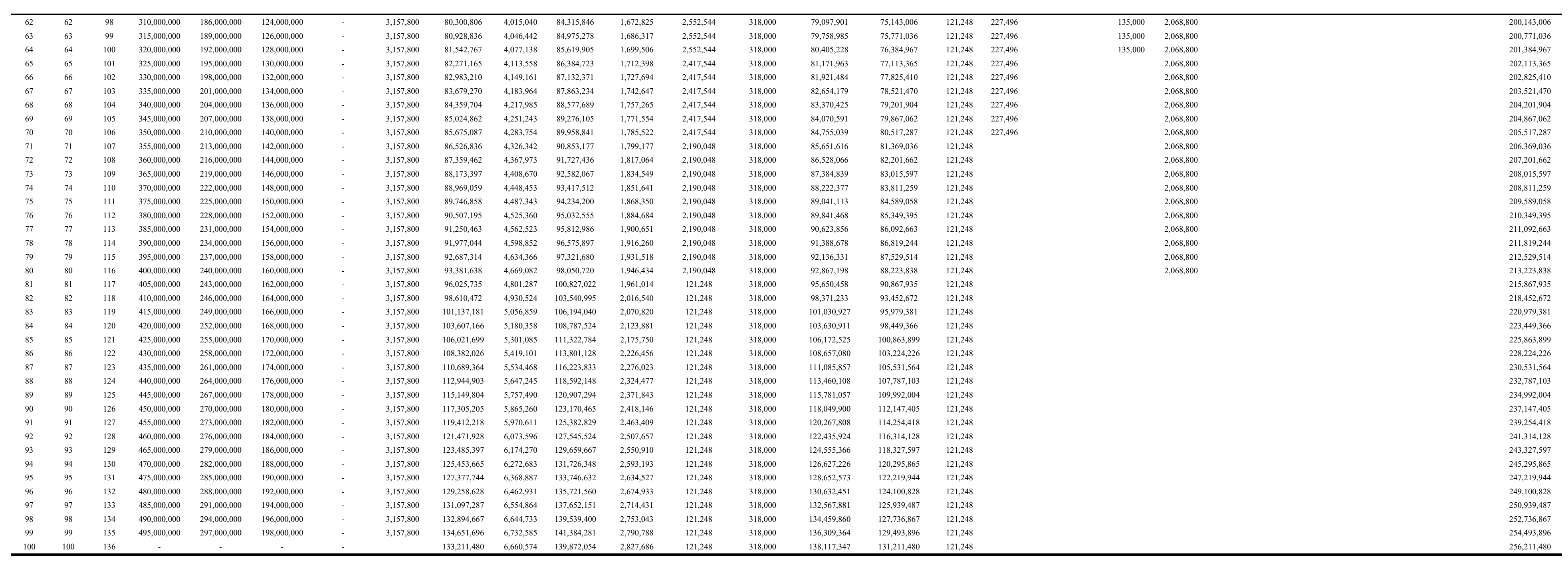


Acknowledgements. We thank to LP2M UIN Sunan Gunung Djati Bandung, which has been providing facilities for research and publication through the BOPTN research fund. Thanks to Annisa Azizah, S.Si has helped directly in the writing of this paper. Thanks were also given to all who has helped directly or indirectly so that these paper can be resolved properly.

\section{References}

[1] W. M. A. W. Ahmad, et.al., "A Case Study of Acceptance Model: A New Integration Model of Education Plan Takaful", International Journal of Advances in Engineering, Science and Technology, Vol. 1, No.2, 2012.

[2] "A Design of Mathematical Modelling for The Mudharabah Scheme in Shariah Insurance", IOP Conference Series: Materials Science and Engineering, Vol.166, 2017.

[3] R. Cahyandari, et.al, "Optimization of Hybrid Model on Hajj Travel", IOP Conference Series: Materials Science and Engineering, Vol. 332, 2018.

[4] R. Cahyandari, et.al, "The Hybrid Model Algorithm on Sharia Insurannce", IOP Conference Series: Journal of Physics: Conf. Series, Vol. 1090, 2018.

[5] P.L. Ghazali, et.al, "Mathematical Modelling in Family Takaful", Journal of Applied Science, 11(19): 3381-3388, 2011.

[6] P.L. Ghazali, et.al, "Implementation of Integration Model for All", Journal of Applied Science Research, 8(3): 1802-1812, 2012.

[7] P.L. Ghazali, et.al, "Integration Model in Premium Life Table of Family Takaful", Journal of Applied Science, 8(7): 3763-3776, 2012.

[8] P.L. Ghazali, et.al, "Comparison of Premium Life Tables between Existing Model and Integration Model in Family Takaful, Journal of Applied Sciences Research, 8(7): 3754-3762, 2012. [9] P.L. Ghazali, et.al, “A Case Study Of Acceptance Model: A New Integration Model of Education Plan Takaful”, International Journal of Advances in Engineering, Science and Technology, 1(2): 169-179, 2012.

[10] P.L. Ghazali, et.al, "Integration Model for Education Plan Takaful: A Case Study for Terengganu, Kelantan, and Perlis, States in Malaysia”, Far East Journal of mathematical sciences, 65(1), 2012

[11] P.L. Ghazali, et.al, "Optimization of Integration Model in Family Takaful", Applied Mathematical Sciences, 9 (39): 1899-1909, 2015.

[12] P.L. Ghazali, et.al, "Integration Model in Auto Takaful Insurance", Far East Journal of Mathematical Sciences, 98(5): 599-611, 2015.

[13] P.L. Ghazali, et.al, " Comparison Mathematical Formulation in Insurance and Takaful", Penerbit Universiti Sultan Zainal Abidin, Kuala Terengganu, 2015.

[14] S. A. Ismail, et.al "Application of Integration Model for Recovery Fund in Takaful Education Plan“, Far East Journal of mathematical sciences, 100(2): 301-313, 2016.

[15] P.L. Ghazali, et.al, "Acceptance of Integrated Modification Model of Auto Takaful Insurance in Malaysia”, Far East Journal of mathematical sciences, 101(8): 1771-1784, 2017.

[16] P.L. Ghazali, et.al, "Medical Integration Model of Family Takaful for Blue Collar", Far East Journal of mathematical sciences, 101(6): 1197, 2017.

[17] P.L. Ghazali, et.al, "Analysis of The Acceptance of Newly Constructed Takaful Education Plan for Learning Disabled Children among Public", International Journal of Academic Research in Business and Social Sciences, 8(11): 1413-1420, 2018.

[18] M. S. Sula, "Sharia Insurance (Life and General) Concepts and Operational Systems (Asuransi Syariah (Life and General) Konsep dan Sistem Operasional)", Jakarta: Gema Insani, 2004.

[19] N. Zawani, "Recovery Model of Takaful Education Plan with Additional Benefit for Parents of Learning Disabled Children", Thesis, Universiti Sultan ZainalAbidin, 2017.

[20] www.Allianz.co.id 